\title{
Actitudes, percepciones y satisfacción de las enfermeras andaluzas con la atención domiciliaria
}

\author{
Antonio Frías Osunaa, M. Ángeles Prieto Rodríguez ${ }^{\text {, }}$, Cristina Heierle Valeroce, \\ Eugenia Gil García d y Carmen Aceijas Hernández \\ aDiplomado en Enfermería y Licenciado en Antropología Social y Cultural. Escuela de Enfermería de la Universidad de J aén. J aén. España. \\ ${ }^{b}$ Diplomada en Enfermería y Licenciada en Ciencias Políticas y Sociología. Escuela Andaluza de Salud Pública de Granada. Granada. \\ 'Diplomada en Enfermería y Licenciada en Enfermería. Escuela de Enfermería Virgen de las Nieves de Granada. Granada. España. \\ ¿Diplomada en Enfermería y Licenciada en Ciencias Políticas y Sociología. E scuela de Enfermería de la Universidad de J aén. J aén. España. \\ eLicenciada en Psicología. Escuela Andaluza de Salud Pública de Granada. Granada. España.
}

\section{Nesumen}

Introducción. El objetivo de este estudio es conocer la satisfacción, las actitudes y las percepciones que las enfermeras andaluzas tienen respecto a la atención domiciliaria, con el propósito de aportar información que oriente las estrategias de cambio y mejora de la calidad de ésta.

Material y métodos. Se trata de un estudio descriptivo transversal mediante cuestionario autoadministrado a profesionales de enfermería que realizan atención domiciliaria en centros de salud de Andalucía. El tamaño de muestra fue de 348 sujetos, con una tasa de respuesta del $61,14 \%$. Las principales mediciones han sido la satisfacción con la atención domiciliaria, las actitudes ante el paciente y familia, la percepción del rol profesional, el locus de control y la percepción sobre la capacitación.

Resultados. EI 71,5\% (intervalo de confianza [IC] del 95\%,65,2-77,8) de las enfermeras están satisfechas con la atención domiciliaria, el 48\% (IC del 95\%, 31-69) se muestran satisfechas con la atención a los pacientes con sida en fase terminal, y el $66 \%$ (IC del $95 \%, 59,1-72,9$ ), el $90 \%$ (IC del $95 \%$, $86,5-94,5)$ y el $65 \%$ (IC del $95 \%, 57,8-72,2$ ) lo están con la atención a pacientes con cáncer, alta precoz y demencia, respectivamente. Entre el 73,53 y el

Investigación financiada por el FIS:98/0653 y por la Consejería de Salud de la J unta de Andalucía: 23/97.

Correspondencia: A. Frías Osuna.

Universidad de J aén. Departamento de Ciencias de la Salud. Área de Enfermería.

Paraje Las Lagunillas. E dificio B-3. 23071 J aén. España.

Correo electrónico: afrias@ujaen.es

Aceptado para su publicación el 16-03-2004.
$80,77 \%$ están de acuerdo con que el papel de la familia es lo más importante para el bienestar del enfermo en pacientes con cáncer, demencia y sida. E I $84,5 \%$ de las enfermeras perciben que se encuentran capacitadas para la prevención/promoción; el 78,5\%, para los aspectos técnicos, y el 77\%, para los aspectos psicosociales.

Conclusi ones. Los niveles de satisfacción de las enfermeras con el trabajo domiciliario son altos. Se valora positivamente la influencia de la familia en el bienestar del enfermo. Entienden que la atención a los enfermos en fase terminal (cáncer y sida) requiere apoyo desde la atención especializada. Dan gran importancia a la coordinación y comunicación entre el equipo de salud para una correcta atención a estos pacientes. Se perciben con mayores necesidades de formación en la atención a los pacientes con sida, así como en los aspectos psicosociales de su práctica profesional.

Palabras clave: Atención domiciliaria. Actitudes profesionales. Satisfacción profesional. Paciente terminal. Demencia. Cirugía ambulatoria. Paciente con sida.

\section{Attitudes, perceptions and satisfaction among Andalusian nurses toward home care}

Introduction. The aim of this study was to identify the attitudes and perceptions of Andalusian nurses toward home care, as well as their satisfaction with this type of care, with the aim of obtaining information that could be used to guide new strategies and improve quality.

Material and methods. We performed a questionnaire-based, cross-sectional descriptive study of nurses working in home care in health centres in Andalusia (Spain). The survey consisted of 
348 questionnaires with a response rate of $61.14 \%$. The main parameters were job satisfaction with home care, attitudes toward patients and their relatives, nurses' perceptions of their professional role and training, and the locus of control.

Results. A total of $71.5 \%(95 \% \mathrm{Cl}, 65.2-77.8)$ of the nurses working in home care felt fulfilled. Forty-eight percent ( $95 \% \mathrm{Cl}, 31-69)$ of nurses working with AIDS patients in the terminal stage, $66 \%(95 \% \mathrm{Cl}, 59.1-72.9)$ of those working with patients with cancer, $90 \%$ ( $95 \%$ $\mathrm{Cl}, 86.5-94.5)$ of those working with early discharged patients and $65 \%(95 \% \mathrm{Cl}, 57.8-72.2)$ of those working with patients with dementia reported job satisfaction. Between $73.53 \%$ and $80.77 \%$ agreed that the role of the family is of the utmost importance for the wellbeing of patients with cancer, mental illness and AIDS. Most nurses (84.5\%) consider themselves competent in prevention campaigns, $78.5 \%$ in technical issues, and $77 \%$ in psycho-social issues.

Conclusi ons. J ob satisfaction among nurses working in home care is high. Family support is highly valued for the well-being of patients. Nurses recognize that the care of patients in the terminal stage (cancer, AIDS) requires specialized care. Coordination and communication between health professionals is important in providing appropriate patient care. Nurses feel they need more training in the care of AIDS patients, as well as in the psychosocial aspects of their profession.

Key words: Home care. Professional attitudes. Professional satisfaction. Terminally ill patient. Dementia. Ambulatory surgery. AIDS patient.

\section{Introducción}

Este estudio es parte del proyecto de investigación "Patrones de Práctica Enfermera en Atención Domiciliaria", que tiene como objetivo general conocer la actividad enfermera en los contextos de la atención en el domicilio. Este ámbito de práctica profesional está recibiendo cada vez más atención y supone una ampliación de la oferta de servicios sanitarios ${ }^{1-6}$.

En un artículo anterior titulado "La práctica enfermera en atención domiciliaria: perfil sociodemográfico y práctica profesional"7, se describen las características demográficas y laborales de las enfermeras que realizan atención domiciliaria en Andalucía, las actividades desarrolladas en el domicilio y las diferencias de actividad profesional según el tipo de paciente atendido.

Con este estudio se pretende conocer qué hacen y cómo piensan las y los enfermeros de atención primaria de Andalucía que realizan atención domiciliaria. Se analiza su práctica profesional ante 4 tipos de pacientes: pacientes con cáncer y sida en fase terminal, an- cianos con demencia y pacientes intervenidos en programas de cirugía mayor ambulatoria (en adelante denominados como "alta precoz"), dado que son los priorizados por el Servicio Andaluz de Salud (SAS) en los contratos-programa en los últimos años ${ }^{8}$.

EI análisis de los patrones de práctica enfermera en la atención domiciliaria se abordó mediante una aproximación al estudio de la conducta humana basado en el Health Belief Model ${ }^{9}$. Este modelo interpretativo basa su explicación de la conducta, en nuestro estudio la práctica enfermera, en tres variables: características personales ( sociodemográficas, conocimientos y habilidades, actitudes, creencias y valores, y factores psicológicos y psicosociales), factores socioculturales y socioambientales, y características de la organización y la estructura. Estas variables pueden explicar la práctica enfermera y su variabilidad. EI modelo se completa, en este proyecto, con las aportaciones de la Teoría del Aprendizaje Social ${ }^{10}$ con respecto a las variables "locus de control" y "autoeficacia". En la figura 1 se expone la estructura teórica del estudio de la práctica enfermera domiciliaria reseñada.

Los diferentes estudios previos que han abordado la satisfacción laboral de las/os enfermeras/os de atención primaria ${ }^{11-18}$ han puesto de manifiesto la existencia de un grado medio de satisfacción general, identificando como elementos que generan mayor nivel de insatisfacción la falta de promoción profesional, la tensión relacionada con el trabajo, la falta de incentivación y el exceso de trabajo.

Este proyecto pretende aportar información para orientar las estrategias de cambio y mejora en la atención domiciliaria en relación con la gestión de las prácticas profesionales, la formación y el reciclaje de las enfermeras y las dinámicas de adaptación y personalización de la oferta de cuidados ante los nuevos retos planteados desde la Administración Sanitaria y condicionados por las actuales necesidades de cuidados ${ }^{19-21}$. Los objetivos específicos que pautan nuestra investigación son: conocer el grado de satisfacción y las actitudes de los profesionales de enfermería con el trabajo en el domicilio, aproximarnos a la percepción que los y las enfermeros/as tienen sobre su preparación ante los nuevos problemas de salud a los que deben dar respuesta, analizar las posibles diferencias en cada uno de los aspectos anteriores en función del tipo de paciente que atienden en el domicilio: pacientes con cáncer, con sida, ancianos con demencia o pacientes en alta precoz.

\section{Sujetos y métodos}

Se ha realizado un estudio descriptivo transver-

sal mediante la utilización de un cuestionario autoadministrado, diseñado y validado específicamente para este estudio ${ }^{22}$. 


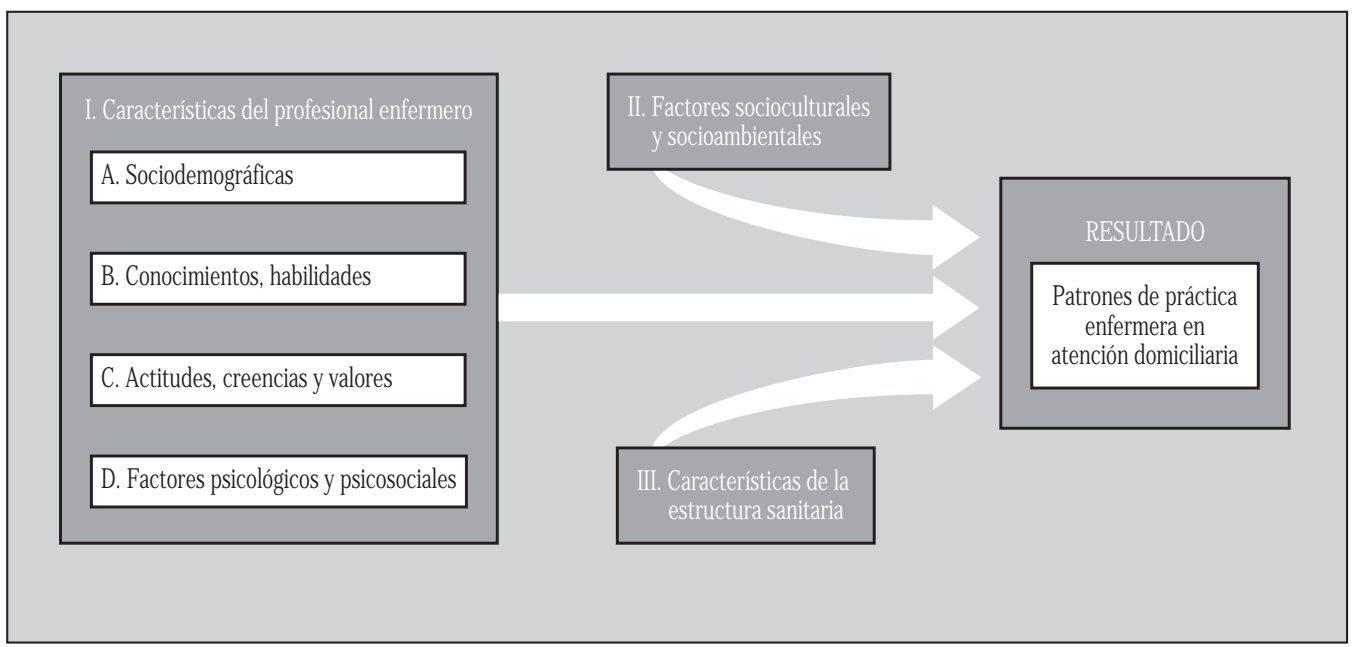

Fig. 1. Factores que influyen en los patrones de práctica enfermera en atención domiciliaria.

El cuestionario consta de 5 bloques de ítems. EI bloque 1 contiene preguntas que describen la actividad domiciliaria (AD) y la actitud de los profesionales ante ella: volumen de pacientes, tiempo dedicado a la $A D$, trabajo en equipo y satisfacción y motivación en el trabajo. Los 4 bloques restantes corresponden a los ítems específicos sobre cada uno de los 4 tipos de pacientes atendidos en el domicilio: pacientes con cáncer, sida, ancianos con demencia y pacientes con alta precoz. Los ítems incluidos en estos 4 bloques son idénticos y se refieren a las actividades desarrolladas en el cuidado, la actitud ante el paciente y la familia, percepción del rol profesional, locus de control, satisfacción profesional, percepción de la capacitación para atender a cada tipo de paciente y necesidades de formación. En la tabla 1 se especifican las dimensiones analizadas en este trabajo.

Las cuestiones sobre las que se preguntó en la escala de capacitación han sido agrupadas en tres componentes bien diferenciados de la práctica enfermera:

- Componente técnico: "diseñar la estrategia de intervención o plan de cuidados", "aplicar tratamientos" y "prevenir complicaciones".

- Componente preventivo/de promoción: "formar e implicar a la familia en los cuidados" y "motivar cambios y promover hábitos de conducta saludables que afecten al estado de salud del paciente".

- Componente psicosocial: "establecer una buena comunicación con el paciente" y "dar respuesta a las reacciones emocionales".

Los sujetos de estudio fueron profesionales de enfermería de equipos de atención primaria de centros de salud de Andalucía que realizaban atención domiciliaria. Para la identificación de sujetos se utilizó la información de la base de datos de personal del SAS.

Se realizó un muestreo polietápico (en cada provincia y, dentro de cada una de ellas, en 2 direcciones: capital y no capital) con afijación proporcional al tamaño de la población enfermera que realizaba atención domiciliaria en cada provincia. El tamaño mínimo propuesto se realizó bajo estimaciones de $p=$ $60 \%$ y un nivel de confianza del $95 \%$, el error admitido fue del 5\%. La muestra mínima necesaria para la realización del estudio resultó ser de 348 sujetos, teniendo en cuenta que el tamaño de la población era de 3.588 enfermeras $/ 0 s^{23}$.

Los cuestionarios fueron enviados por vía postal con sobres nominales a la atención de la persona que debía cumplimentarlos. La recogida se hizo en mano por personas contratadas específicamente para ello.

Los datos se han analizado, en primer lugar, mediante análisis descriptivo básico. El cálculo de los intervalos de confianza de las proporciones se ha realizado utilizando la fórmula propuesta por Magallón y Antoñanzas ${ }^{24}$. A continuación se calcularon el contraste de medias, el análisis de la varianza y de contingencia (dependiendo del nivel de medida de la variable). Finalmente, al objeto de determinar las posibles diferencias de las actitudes, satisfacción y percepción por parte de las enfermeras según el tipo de paciente atendido, se ha realizado un análisis estadístico de comparación de medidas repetidas dentro del modelo lineal general; esta prueba informa

\section{TABLA 1. Dimensiones analizadas en este estudio}

Satisfacción global con su trabajo en el centro de salud y con la atención domiciliaria

Satisfacción con el trabajo en el domicilio en función del tipo de paciente: con cáncer, sida, ancianos con demencia y en alta precoz

Actitud ante la familia del paciente

Actitud ante cada tipo de paciente: con cáncer, sida, ancianos con demencia y en alta precoz

Percepción del rol profesional

Locus de control

Percepción sobre la capacitación

Percepción sobre la formación recibida 
TABLA 2. Actitudes de las enfermeras ante la familia del paciente atendido en el domicilio

\begin{tabular}{|c|c|c|c|c|c|c|c|c|}
\hline \multirow{2}{*}{ Tipo de paciente } & \multicolumn{2}{|c|}{ Cáncer } & \multicolumn{2}{|c|}{ Alta precoz } & \multicolumn{2}{|c|}{ Demencia } & \multicolumn{2}{|c|}{ Sida } \\
\hline & Frecuencia & $\%$ & Frecuencia & $\%$ & F recuencia & $\%$ & Frecuencia & $\%$ \\
\hline \multicolumn{9}{|c|}{ "En general, la familia de estos pacientes se implica positivamente en los cuidados": } \\
\hline Alqún nivel de desacuerdo & 22 & 12 & 38 & 21,6 & 45 & 26,5 & 10 & 29 \\
\hline Indiferente & 31 & 17 & 20 & 11,4 & 34 & 20 & 7 & 21 \\
\hline Algún nivel de acuerdo & 130 & 71 & 118 & 67 & 91 & 53,5 & 17 & 50 \\
\hline Total & 183 & 100 & 176 & 100 & 170 & 100 & 34 & 100 \\
\hline \multicolumn{9}{|c|}{ "El papel de la familia es lo más importante para el bienestar del enfermo": } \\
\hline Nada de acuerdo & 3 & 1,6 & 21 & 12 & 5 & 3 & 2 & 6 \\
\hline Indiferente & 10 & 5,4 & 29 & 16 & 7 & 4 & 1 & 3 \\
\hline Algo de acuerdo & 169 & 93 & 127 & 72 & 158 & 93 & 31 & 91 \\
\hline Total & 182 & 100 & 177 & 100 & 170 & 100 & 34 & 100 \\
\hline
\end{tabular}

sobre la variabilidad en las respuestas en función del tipo de paciente atendido.

\section{Resultados}

Tasa de respuesta

La tasa de respuesta fue del $61,14 \%$. En todas las provincias se superó el $50 \%$ de respuestas excepto en Sevilla, donde se consiguió sólo el $44 \%$. En Huelva se alcanzó una tasa de respuesta del 90,91\% 7 .

\section{Características sociodemográficas}

De las 203 personas que respondieron al cuestionario, 133 eran mujeres (65,5\%). La edad media fue de 39,72 \pm 7,66 años. Más de la mitad de la muestra estaba comprendida entre 35 y 44 años. Como media, llevaban trabajando en atención primaria 10,03 años. La mitad de ellas eran estatutarias y la otra mitad eran contratadas o interinas.

\section{Satisfacción laboral}

El 86\% (intervalo de confianza [IC] del 95\%, 81,2$90,8)$ de las enfermeras se encuentran bastante 0 muy de acuerdo con la afirmación: "En general me siento satisfecho/a de trabajar en atención primaria", y el $71,5 \%$ (IC del 95\%, 65,7-77,8) lo están con la afir- mación: "En general me siento satisfecho/a de trabajar en atención domiciliaria". Respecto a la satisfacción con diferentes aspectos del trabajo domiciliario, destaca que el $52 \%$ (IC del $95 \%, 45,1-58,9$ ) se encuentra insatisfecho con la facilidad de desplazamiento a los domicilios. En el resto de aspectos -coordinación con el médico, coordinación con el resto del equipo, apoyo del adjunto de enfermería y con la información recibida-, el 50\% (IC del 95\%, 43,1-56,9), el $58 \%$ (IC del $95 \%, 51,2-64,8$ ), el $84 \%$ (IC del $95 \%$, $79-89$ ) y el $61 \%$ (IC del $95 \%, 54,2-67,8$ ) respectivamente, se sitúan en la zona de satisfacción.

La mayoría de las enfermeras se sitúa en la zona que indica satisfacción con la atención domiciliaria de los pacientes con cáncer, con alta precoz y con demencia ( $66 \%$; IC del $95 \%, 59,1-72,9)$, el $90 \%$ (IC del $95 \%, 86,5-94,5)$ y el $65 \%$ (IC del $95 \%, 57,8$ $72,2)$, respectivamente. En el caso de la atención a pacientes con sida en fase terminal, el $48 \%$ (IC del $95 \%, 31-69$ ) de los que responden a la encuesta muestra algún nivel de satisfacción.

Actitud ante la familia y el paciente

Ante la afirmación: "En general, la familia de estos pacientes se implica positivamente en los cuidados", en pacientes de cáncer, alta precoz y demencia, el

TABLA 3. Actitudes de las enfermeras ante el tipo de paciente a atender en el domicilio

\begin{tabular}{|c|c|c|c|c|c|c|c|c|}
\hline \multirow{2}{*}{ Tipo de paciente } & \multicolumn{2}{|c|}{ Cáncer } & \multicolumn{2}{|c|}{ Alta precoz } & \multicolumn{2}{|c|}{ Demencia } & \multicolumn{2}{|l|}{ Sida } \\
\hline & Frecuencia & $\%$ & Frecuencia & $\%$ & F recuencia & $\%$ & Frecuencia & $\%$ \\
\hline \multicolumn{9}{|c|}{ "Si pudiera elegir qué tipo de paciente atender en atención domiciliaria, elegiría este tipo": } \\
\hline Algún nivel de desacuerdo & 69 & 38 & 27 & 15,5 & 57 & 33,5 & 17 & 50 \\
\hline Indiferente & 48 & 26,3 & 23 & 13 & 41 & 24,1 & 7 & 20,6 \\
\hline Algún nivel de acuerdo & 65 & 35,7 & 125 & 71,5 & 72 & 42,4 & 10 & 29,4 \\
\hline Total & 182 & 100 & 175 & 100 & 170 & 100 & 34 & 100 \\
\hline \multicolumn{9}{|c|}{$\begin{array}{l}\text { "Con frecuencia aparecen dificultades al atender a este tipo de pacientes que requieren atención especializada } \\
\text { que yo no puedo dar en el domicilio": }\end{array}$} \\
\hline Algún nivel de desacuerdo & 51 & 28 & 62 & 35 & 62 & 37 & 8 & 23,5 \\
\hline Indiferente & 19 & 10,3 & 34 & 19,2 & 27 & 16 & 3 & 8,5 \\
\hline Algún nivel de acuerdo & 113 & 61,7 & 81 & 45,8 & 80 & 47 & 23 & 68 \\
\hline Total & 183 & 100 & 177 & 100 & 169 & 100 & 34 & 100 \\
\hline
\end{tabular}


TABLA 4. Percepción de las enfermeras del rol profesional en atención domiciliaria

\begin{tabular}{|c|c|c|c|c|c|c|c|c|}
\hline \multirow{2}{*}{ Tipo de paciente } & \multicolumn{2}{|c|}{ Cáncer } & \multicolumn{2}{|c|}{ Alta precoz } & \multicolumn{2}{|c|}{ Demencia } & \multicolumn{2}{|c|}{ Sida } \\
\hline & Frecuencia & $\%$ & Frecuencia & $\%$ & Frecuencia & $\%$ & Frecuencia & $\%$ \\
\hline \multicolumn{9}{|c|}{ "La valoración de la problemática social es parte de mi labor": } \\
\hline Algún nivel de desacuerdo & 23 & 12,6 & 37 & 21 & 13 & 7,6 & 1 & 3 \\
\hline Indiferente & 22 & 12 & 29 & 16,3 & 31 & 18,3 & 6 & 17,6 \\
\hline Algún nivel de acuerdo & 138 & 75,4 & 111 & 62,7 & 126 & 74,1 & 9 & 79,4 \\
\hline Total & 183 & 100 & 177 & 100 & 170 & $100^{\prime 2}$ & 34 & 100 \\
\hline \multicolumn{9}{|c|}{ "Dar respuesta a los problemas emocionales del paciente o de la familia es parte de mi trabajo": } \\
\hline Algún nivel de desacuerdo & 6 & 3,3 & 14 & 8 & 5 & 3 & 2 & 5,9 \\
\hline Indiferente & 12 & 6,5 & 22 & 12,4 & 8 & 4,7 & 4 & 11,8 \\
\hline Algún nivel de acuerdo & 165 & 90,2 & 141 & 79,6 & 157 & 92,3 & 28 & 82,3 \\
\hline Total & 183 & $100^{2}$ & 177 & 100 & 170 & 100 & 34 & 100 \\
\hline \multicolumn{9}{|c|}{ "Si quiero prestar una atención de calidad en el domicilio, mi trabajo debe centrarse en aliviar los síntomas de la enfermedad": } \\
\hline Algún nivel de desacuerdo & 75 & 42 & 62 & 35 & 72 & 42,4 & 13 & 38,2 \\
\hline Indiferente & 26 & 14,4 & 27 & 16 & 34 & 20 & 6 & 17,7 \\
\hline Algún nivel de acuerdo & 79 & 43,6 & 85 & 49 & 64 & 37,6 & 15 & 44,1 \\
\hline Total & 180 & 100 & 174 & 100 & 170 & 100 & 34 & 100 \\
\hline
\end{tabular}

$71 \%$ (IC del $95 \%, 64,4-77,6$ ), el $67 \%$ (IC del 95\%,60$73,9)$ y el $53,5 \%$ (IC del $95 \%, 46-60,9)$ de los encuestados muestran, respectivamente, al gún nivel de acuerdo con esta afirmación, en el $50 \%$ (IC del 95\%, 33,2-66,8) para el caso de pacientes con sida. Con la afirmación: "E I papel de la familia es lo más importante para el bienestar del enfermo", se muestran de acuerdo el $93 \%$ (IC del 95\%, 89,4-96,6) en el caso de pacientes con cáncer, el $72 \%$ (IC del $95 \%$, 65,4-78,6) en pacientes de alta precoz, el 93\% (IC del $95 \%, 89,2-96,8)$ en el caso de los pacientes con demencia y el $91 \%$ (IC del $95 \%, 81,4-100)$ en pacientes con sida (tabla 2 ).

Ante la afirmación: "Si pudiera elegir qué tipo de pacientes atender en atención domiciliaria, elegiría atender este tipo", el 71\% (IC del 95\%, 64,3-77,7) muestra algún nivel de acuerdo en el caso de pacientes de alta precoz, y sólo el 36\% (IC del 95\%, 29-43), el $42 \%$ (IC del $95 \%, 34,6-49,4$ ) y el $29 \%$ (IC del $95 \%$, 13,7-44,3) para pacientes de cáncer, demencia y sida, respectivamente. Cuando a las enfermeras se les plantea como afirmación: "Con frecuencia aparecen dificultades al atender a este tipo de pacientes que requieren atención especializada que yo no puedo dar en el domicilio", en el caso de cáncer en fase terminal, el $62 \%$ (IC del $95 \%, 55-69$ ) de ellas se sitúa en la zona de acuerdo, y en el caso de sida el $68 \%$ (IC del $95 \%$, $51,2-82,8$ ), en pacientes de alta precoz el $45 \%$ (IC del $95 \%, 37,7-52,3$ ) y el $47 \%$ (IC del $95 \%, 39,5-54,5)$ en el caso de pacientes con demencia (tabla 3 ).

\section{Percepción del rol profesional}

Ante la cuestión: "La valoración de la problemática social es parte de mi labor", en los cuatro tipos de pacientes la mayoría de los encuestados se muestran de acuerdo, el 75,4\% (IC del 95\%, 69,2-81,6) para pacientes de cáncer, el $62,7 \%$ (IC del $95 \%, 55,6-69,8)$ en el caso de alta precoz, el 74,1\% (IC del 95\%, 67,6-80,6) en la demencia y el 79,4\% (IC del 95\%,65,9-92,9) en el sida. Con la afirmación: "Dar respuesta a los problemas emocionales del paciente 0 de la familia es parte de mi trabajo", manifiestan su acuerdo el 90,2\% (IC del $95 \%, 85,9-94,5)$ en el caso de los pacientes con cáncer terminal, el 79,6\% (IC del 95\%, 73,7-85,5) para el alta precoz, el 92,3\% (IC del 95\%, 88,3-96,3) para la demencia y el $82,3 \%$ (IC del $95 \%, 69,5-95,1$ ) para pacientes con sida terminal (tabla 4).

Ante la afirmación: "Si quiero prestar una atención de calidad en el domicilio, mi trabajo debe centrarse en aliviar los síntomas de la enfermedad", en el caso de pacientes con cáncer en fase terminal, el $44 \%$ (IC del 95\%, 36,8-51,2) de los encuestados está de acuerdo con la frase, el $49 \%$ (IC del 95\%, 41,6$56,4)$ en el caso de pacientes en alta precoz, el $38 \%$ (IC del $95 \%, 30,7-45,3)$ en los ancianos con demencia y el $44 \%$ (IC del 95\%, 27,3-60,7) en el caso de los pacientes con sida en fase terminal (tabla 4).

\section{Locus de control}

Ante la afirmación: "A veces tengo la sensación de que nunca logro cumplir los objetivos que me planteo con estos pacientes", el 52\% (IC del 95\%, 44,8-59,2) está de acuerdo con ella para el caso de pacientes con cáncer, el 16,3\% (IC del 95\%, 10,9-21,7), el 58,2\% (IC del $95 \%, 50,8-65,6$ ) y el $55 \%$ (IC del $95 \%, 38,3-71,7$ ) en el caso de pacientes de alta precoz, demencia y sida, respectivamente. EI $58 \%$ (IC del 95\%, 50,8-65,2) en el caso de pacientes con cáncer, el $26 \%$ (IC del $95 \%, 19,6-32,4$ ) en el alta precoz, el $59 \%$ (IC del $95 \%$, $51,6-66,4)$ en la demencia y el $60 \%$ (IC del $95 \%$, 4575 ) en pacientes con sida en fase terminal muestran algún nivel de acuerdo con la afirmación: "Cuidar a este tipo de pacientes supone demandas de tiempo extra del que no dispongo" ( tabla 5).

El porcentaje de encuestados que se sitúa en la zona de acuerdo con la afirmación: "La evolución sa- 
TABLA 5. Locus de control

\begin{tabular}{|c|c|c|c|c|c|c|c|c|}
\hline \multirow{2}{*}{ Tipo de paciente } & \multicolumn{2}{|c|}{ Cáncer } & \multicolumn{2}{|c|}{ Alta precoz } & \multicolumn{2}{|c|}{ Demencia } & \multicolumn{2}{|c|}{ Sida } \\
\hline & Frecuencia & $\%$ & Frecuencia & $\%$ & Frecuencia & $\%$ & Frecuencia & $\%$ \\
\hline \multicolumn{9}{|c|}{ "A veces tengo la sensación de que nunca logro cumplir los objetivos que me planteo con estos pacientes": } \\
\hline Algún nivel de desacuerdo & 68 & 37 & 134 & 75,7 & 47 & 27,6 & 8 & 24,2 \\
\hline Indiferente & 20 & 11 & 14 & 8 & 26 & 15,2 & 7 & 21,2 \\
\hline Algún nivel de acuerdo & 95 & 52 & 29 & 16,3 & 100 & 58,2 & 18 & 54,6 \\
\hline Total & 183 & 100 & 177 & 100 & 170 & 100 & 33 & 100 \\
\hline \multicolumn{9}{|c|}{ "Cuidar a este tipo de pacientes supone demandas de tiempo extra del que no dispongo": } \\
\hline Algún nivel de desacuerdo & 57 & 31 & 105 & 59,3 & 46 & 27 & 12 & 36,4 \\
\hline Indiferente & 20 & 11 & 25 & 14,2 & 24 & 14 & 1 & 3 \\
\hline el de acuerdo & 106 & 58 & 47 & 26,5 & 100 & 59 & 20 & 60,6 \\
\hline Total & 183 & 100 & 177 & 100 & 170 & 100 & 33 & 100 \\
\hline \multicolumn{9}{|c|}{ "La evolución satisfactoria del paciente depende, en gran medida, de la coordinación y comunicación del equipo que le atiende": } \\
\hline Algún nivel de desacuerdo & 13 & 7,1 & 12 & 6,8 & 13 & 7,6 & 4 & 12 \\
\hline Indif & 10 & 5,5 & 10 & 5,6 & 11 & 6,4 & 3 & 9 \\
\hline de acuerdo & 160 & 87,4 & 155 & 87,6 & 146 & 86 & 26 & 79 \\
\hline \multirow{2}{*}{\multicolumn{9}{|c|}{ "La calidad de vida de estos pacientes depende, en gran medida, de las habilidades y conocimientos del profesional de enfermería" }} \\
\hline & & & & & & & & \\
\hline Algún nivel de desacuerdo & 18 & 9,8 & 16 & 9 & 21 & 12,4 & 8 & 24,2 \\
\hline Indifer & 36 & 19,7 & 17 & 9,60 & 28 & 16,47 & 4 & 12,2 \\
\hline el de acuerdo & 129 & 70,5 & 144 & 81,4 & 121 & 71,2 & 21 & 63,6 \\
\hline Total & 183 & 100 & 177 & 100 & 170 & 100 & 33 & 100 \\
\hline \multicolumn{9}{|c|}{ "No importa que lo que haga en las fases avanzadas de la enfermedad. Si el paciente ha de empeorar, empeorará": } \\
\hline Nada de acuerdo & 128 & 70,3 & & & 117 & 69,2 & 17 & 53 \\
\hline Indiferente & 12 & 6,6 & & & 21 & 12,4 & 5 & 15,7 \\
\hline Algo de acuerdo & 42 & 23,1 & & & 31 & 18,4 & 10 & 31,3 \\
\hline Total & 182 & 100 & & & 169 & 100 & 32 & 100 \\
\hline
\end{tabular}

tisfactoria del paciente depende, en gran medida, de la coordinación y comunicación del equipo que le atiende" es del $87 \%$ (IC del 95\%, 82,1-91,9) en el caso de pacientes con cáncer, del $88 \%$ (IC del $95 \%$, $83,2-92,8$ ) en pacientes con alta precoz, del $86 \%$ (IC del $95 \%, 80,8-91,2)$ en pacientes con demencia y del 79\% (IC del 95\%, 64-92) en pacientes con sida. EI $70 \%$ (IC del 95\%, 63,4-76,6) de las enfermeras en- cuestadas en el caso de pacientes con cáncer, el $81 \%$ (IC del 95\%, 75,2-86,8) en pacientes con alta precoz, el $71 \%$ (IC del 95\%, 64,2-77,8) en pacientes con demencias y el $64 \%$ (IC del $95 \%, 47,9-80,1$ ) en pacientes con sida se muestra de acuerdo, en alguna medida, con la afirmación: "La calidad de vida de estos pacientes depende de las habilidades y conocimientos del profesional de enfermería". En el caso de pa-

TABLA 6. Percepción de las enfermeras de su nivel de capacitación según el componente "técnico", "preventivo/de promoción" y "psicosocial"

\begin{tabular}{|c|c|c|c|c|c|c|c|c|}
\hline \multirow{2}{*}{ Tipo de paciente } & \multicolumn{2}{|c|}{ Cáncer } & \multicolumn{2}{|c|}{ Alta precoz } & \multicolumn{2}{|c|}{ Demencia } & \multicolumn{2}{|c|}{ Sida } \\
\hline & Frecuencia & $\%$ & Frecuencia & $\%$ & Frecuencia & $\%$ & Frecuencia & $\%$ \\
\hline \multicolumn{9}{|c|}{ "Capacitación técnica percibida": } \\
\hline \multicolumn{3}{|c|}{1 (nada capacitado/a) a 2,9 } & - & & - & & - & - \\
\hline \multicolumn{2}{|l|}{$\begin{array}{l}3,0 \text { a } 4,9 \\
5,0 \text { a } 7 \text { (plenamente }\end{array}$} & 19,13 & 14 & 7,91 & 38 & 22,35 & 12 & 36,36 \\
\hline \multicolumn{2}{|l|}{ capacitado/a) } & 80,33 & 163 & 92,09 & 132 & 77,65 & 21 & 63,63 \\
\hline \multirow{2}{*}{\multicolumn{3}{|c|}{$\begin{array}{l}183 \\
\text { "Total } \\
\text { "Capacitación preventiva/de promoción percibida”: }\end{array}$}} & 177 & 100 & 170 & 100 & 33 & 100 \\
\hline & & & \multicolumn{6}{|c|}{ "Capacitación preventiva/de promoción percibida": } \\
\hline \multicolumn{2}{|c|}{1 (nada capacitado/a) a 2,9 } & - & - & - & - & - & 2 & 6,06 \\
\hline \multicolumn{2}{|l|}{$\begin{array}{l}3,0 \text { a } 4,9 \\
5,0 \text { a } 7 \text { (plenamente }\end{array}$} & 11,48 & 12 & 6,82 & 33 & 19,41 & 8 & 24,24 \\
\hline \multicolumn{2}{|l|}{ capacitado/a) } & 88,52 & 164 & 93,18 & 137 & 80,59 & 23 & 69,70 \\
\hline \multirow{2}{*}{\multicolumn{9}{|c|}{ "Capacitación psicosocial percibida": }} \\
\hline & & & & & & & & \\
\hline \multirow{2}{*}{$\begin{array}{l}1 \text { ( nada capacitado/a) a 2,9 } \\
3,0 \text { a } 4,9\end{array}$} & - & \multicolumn{2}{|c|}{$\begin{array}{l}\text { “Capacitación psicosocial percibida”: } \\
1 \text { (nada capacitado/a) a } 29\end{array}$} & - & 9 & 5,33 & 3 & 9,09 \\
\hline & 41 & 22,40 & 12 & 6,78 & 57 & 33,73 & 5 & 15,15 \\
\hline capacitado/a) & 142 & 77,60 & 165 & 93,22 & 103 & 60,95 & 25 & 75,75 \\
\hline Total & 183 & 100 & 177 & 100 & 169 & 100 & 33 & 100 \\
\hline
\end{tabular}


TABLA 7. Percepción de las enfermeras de la formación recibida para la atención domiciliaria

\begin{tabular}{|c|c|c|c|c|c|c|c|c|}
\hline \multirow{2}{*}{ Tipo de paciente } & \multicolumn{2}{|c|}{ Cáncer } & \multicolumn{2}{|c|}{ Alta precoz } & \multicolumn{2}{|c|}{ Demencia } & \multicolumn{2}{|c|}{ Sida } \\
\hline & Frecuencia & $\%$ & Frecuencia & $\%$ & Frecuencia & $\%$ & Frecuencia & $\%$ \\
\hline \multicolumn{9}{|c|}{ “La formación que he recibido es suficiente para dar respuesta específica a los problemas que plantean este tipo de pacientes”: } \\
\hline Nada de acuerdo & 71 & 38,8 & 26 & 14,7 & 60 & 35,3 & 17 & 53 \\
\hline Indiferente & 34 & 18,6 & 10 & 5,3 & 39 & 22,9 & 5 & 15,7 \\
\hline Algo de acuerdo & 78 & 42,6 & 141 & 80 & 71 & 41,8 & 10 & 31,3 \\
\hline Total & 183 & 100 & 177 & 100 & 170 & 100 & 32 & 100 \\
\hline
\end{tabular}

cientes con cáncer y demencia, alrededor del $70 \%$ se muestra en desacuerdo con la afirmación: "No importa lo que haga en las fases avanzadas de la enfermedad. Si el paciente ha de empeorar, empeorará", de lo que se desprende que la mayoría de las enfermeras percibe que la evolución del paciente depende de su intervención. Para los pacientes de sida, el $31 \%$ (IC del 95\%, 15,5-46,5) de las enfermeras está de acuerdo con que, hagan lo que hagan, si el paciente ha de empeorar, empeora (tabla 5).

\section{Percepción sobre la capacitación}

En la tabla 6 se muestran los datos que hacen referencia a la percepción del nivel de capacitación según cada uno de estos componentes. El 92\% (IC del $95 \%, 88-96)$ de las enfermeras se percibe con capacitación (niveles entre el 5 y el 7 de la escala) en el "componente técnico" para la atención a los pacientes de alta precoz, el 80\% (IC del 95\%, 74,2-85,8) para la atención de pacientes con cáncer y el $78 \%$ (IC del 95\%, 71,8-84,2) para la atención a ancianos con demencia. EI $64 \%$ (IC del 95\%, 47,9-80,1) se siente capacitado en el caso de los pacientes con sida. Para las actividades relacionadas con la prevención y la promoción, el 93,18\% (IC del 95\%, 89,4896,88 ) se percibe con capacitación (niveles entre el 5 y el 7 de la escala) para atender a pacientes de alta precoz, el 88\% (IC del 95\%, 82,1-91,9) para atender a los pacientes con cáncer terminal, el $81 \%$ (IC del $95 \%, 75,1-86,9)$ para atender a ancianos con demencia y el 70\% (IC del 95\%, 54,6-85,4) en el caso de pacientes con sida en fase terminal. EI 93\% (IC del $95 \%, 89,3-96,7)$ de las enfermeras encuestadas se siente capacitado ( se sitúan en los niveles mayores de la escala, entre 5 y 7) para la atención a pacientes de alta precoz en el desarrollo de las actividades incluidas en el componente psicosocial, con el $78 \%$ (IC del $95 \%, 72-84$ ), el $76 \%$ (IC del $95 \%, 69,6-82,4$ ) y el $61 \%$ (IC del 95\%, 44,6-77,4) en el caso de pacientes con cáncer, pacientes con sida y ancianos con demencia, respectivamente. EI mayor porcentaje de enfermeras con percepción de capacitación (valores entre el 5 y el 7 en la escala) se sitúa en el componente "preventivo/de promoción" (entre un $69,70 \%$ en el caso de pacientes con sida y un $93,18 \%$ en el caso de alta precoz), seguido del componente "téc- nico" (entre un $63,63 \%$ en el caso de pacientes con sida y un $92,09 \%$ en el caso de pacientes con alta precoz) y del componente "psicosocial" (entre un $60,95 \%$ en el caso de pacientes con demencia y un $93,22 \%$ en el caso de alta precoz) (tabla 6).

\section{Formación recibida}

Respecto a la opinión de las enfermeras sobre la formación recibida para dar respuesta específica a los problemas que plantean estos pacientes, el $80 \%$ (IC del 95\%, 74,1-85,9) está de acuerdo con que la formación recibida es suficiente en el caso de los pacientes de alta precoz, con el $43 \%$ (IC del 95\%, 35,850,2 ), el $42 \%$ (IC del $95 \%, 34,6-49,4$ ) y el $31 \%$ (IC del $95 \%, 15,5-46,5)$ para pacientes con cáncer, demencia y sida, respectivamente ( tabla 7).

Los datos referidos a la pregunta: “¿Hay diferencias en las actitudes y opiniones de las enfermeras en función del tipo de paciente al que atienden en el domicilio?", señalamos que el grupo de pacientes con sida fue excluido por la escasa tasa de respuestas.

Excepto en tres casos ( "La evolución satisfactoria del paciente depende, en gran medida, de la coordinación y comunicación del equipo que le atendemos", "La calidad de vida de estos pacientes depende, en gran medida, de las habilidades y conocimientos del profesional de enfermería" y "No importa lo que haga en las fases avanzadas de la enfermedad. Si el paciente ha de empeorar, empeorará"), el análisis resultó positivo: se observaron diferencias estadísticamente significativas entre las respuestas que se dieron a las preguntas en función del problema de salud atendido en el ámbito de atención domiciliaria $(p<0,05)$. Sin embargo, al eliminar del análisis las respuestas referentes a la atención de pacientes en alta precoz, por tratarse de un tipo de enfermos con necesidades de cuidados muy diferentes de los otros dos ( cáncer y ancianos con demencia), la variabilidad es menor.

Los resultados muestran que hay diferencias significativas $(p<0,05)$ en las respuestas de los encuestados en función del problema de salud atendido (cáncer en fase terminal o demencia en fase terminal) para los ítems:

- Implicación positiva de la familia en los cuidados del paciente. 
- Dar respuesta a los problemas emocionales como parte del rol profesional.

- Aparición frecuente de dificultades en la atención a estos pacientes que no pueden ser atendidos desde la atención domiciliaria al requerir una intervención especializada.

- Capacitación en la aplicación de tratamientos.

- Capacitación para formar e implicar a la familia.

- Capacitación para establecer una buena comunicación con el paciente.

- Capacitación para dar respuesta a las reacciones emocionales.

Para el resto de los ítems, las diferencias en las respuestas no parecen depender de que el paciente atendido en el domicilio sea oncológico o anciano con demencia.

\section{Discusión}

La tasa de respuesta media obtenida es similar a

la que se obtiene en estudios de este tipo $0^{11-14,16-18,25}$. Las diferencias en las tasas de respuesta entre las diferentes provincias deben estar motivadas por la dificultad de localización de los profesionales relacionada con la calidad de los datos disponibles. No se observan diferencias en las características sociodemográficas entre los enfermos que responden de las diferentes provincias.

En el caso de la atención a pacientes con sida en fase terminal, sólo 34 de los encuestados estaban atendiendo o habían atendido en los últimos 3 años a pacientes de estas características, lo que condiciona y limita la interpretación de los datos y su inferencia al resto de la población.

Los niveles de satisfacción con el trabajo son altos, tanto en relación con el desempeño del trabajo en atención primaria como con la atención domiciliaria, en contraste con otros estudios previos que identificaban un nivel de satisfacción medioni-18. El elemento que provoca una mayor insatisfacción es la dificultad para el desplazamiento al domicilio del paciente. La coordinación con el resto del equipo y con el médico alcanza niveles medios de satisfacción, lo que coincide en cierta medida con el estudio de Benítez et $a^{26}$, en el que se identifican, como una de las dificultades para la realización de cuidados paliativos en atención primaria, "Ios problemas de coordinación en la organización interna de los equipos".

La práctica totalidad de las enfermeras (90\%) se encuentran satisfechas con la atención a los pacientes de alta precoz; la atención a los pacientes con sida en fase terminal es la que proporciona satisfacción en un menor número de enfermeras. Probablemente, esta situación responda a diferentes condicionantes, entre los que podríamos destacar el pronóstico de cada tipo de paciente, la efectividad de los cuidados en la recu- peración, las características socioeconómicas a las que pertenece, mayoritariamente, cada grupo de pacientes, la consideración social hacia cierto tipo de conductas relacionadas con la transmisión del sida, etc. La confirmación de las causas de estas diferencias en el grado de satisfacción según el tipo de paciente requiere la realización de estudios más específicos que nos permitan explorar con más profundidad este ámbito del estudio.

La evaluación de las enfermeras a la implicación de la familia en los cuidados a estos pacientes, si bien apuntan hacia una valoración positiva, no es del todo clara. Las puntuaciones más elevadas se alcanzan en los puntos medios de la escala, "algo de acuerdo", lo que indica que no hay una satisfacción clara con la implicación de la familia en los cuidados de estos pacientes. Llama especialmente la atención la cantidad de casos que se sitúan en la categoría de "indiferente". Sin embargo, la familia es valorada por la mayoría de las enfermeras como una potente fuente de bienestar para el enfermo y no identifican su presencia en la habitación como algo que dificulte su trabajo, sino todo lo contrario.

La mayoría de las enfermeras muestra una voluntad favorable para la atención de este tipo de pacientes y si pudiera elegir el tipo de enfermo a atender preferiría al de alta precoz, seguido de los pacientes con demencia y cáncer. Sólo en el caso de pacientes con sida, el $50 \%$ de las enfermeras elegiría a otro tipo de paciente. Este dato contrasta con los resultados encontrados por Suárez-Varela et al ${ }^{27}$, en los que sólo el $21 \%$ de los médicos de su estudio, si tuvieran la posibilidad, no trabajaría con estos pacientes. Estas diferencias pueden ser debidas a que, en el caso de las enfermeras, nos referimos a pacientes en fase terminal, y en el caso de los médicos, el estudio se centra en pacientes con infección por el virus de la inmunodeficiencia humana (VIH)/sida. Esta situación se puede explicar parcialmente por las posibles barreras de comunicación con este tipo de pacientes asociadas a la carencia de habilidades específicas del sanitario ${ }^{28}$.

En la atención a estos pacientes, en el caso de pacientes con cáncer en fase terminal y sida, la mayoría de las enfermeras encuentra dificultades que, según ellas, requieren atención especializada, lo que no ocurre en los pacientes con demencia y alta precoz.

En cuanto a la percepción del rol profesional, la mayoría de las enfermeras entiende que la valoración de la problemática social y dar respuestas a los problemas emocionales del paciente y familia es parte de su trabajo en atención domiciliaria. No hay este mismo nivel de acuerdo cuando el rol profesional se centra en aliviar los síntomas de la enfermedad. Como podemos observar, la distribución de las respuestas presenta una alta variabilidad, lo que pone de 
manifiesto la existencia de patrones de práctica muy diferentes respecto a esta cuestión. Parece haber un mayor nivel de acuerdo con centrarse en aliviar los síntomas de la enfermedad en el caso de pacientes con alta precoz, cáncer y sida terminal.

E stos hechos, junto con las actividades desarrolladas en atención domiciliaria, parecen estar apuntando hacia un nuevo enfoque de la actividad profesional, más de acorde con las necesidades de cuidados de este tipo de usuarios.

E n la evolución del paciente, las enfermeras dan gran importancia a la coordinación y comunicación con el equipo de salud. Esto pone de manifiesto, por una parte, el elevado valor que éstas otorgan al trabajo en equipo y, por otra, la marcada influencia que las enfermeras atribuyen a los factores externos a su propia práctica. Del mismo modo, valoran de forma importante la influencia de sus conocimientos y habilidades sobre la calidad de vida y la evolución de estos pacientes.

EI número de enfermeras que se sienten capacitadas para la atención a los pacientes con alta precoz es el más elevado, seguido de la atención a pacientes con cáncer, demencia y, en menor número, las que se sienten capacitadas para la atención al paciente con sida. Esta percepción sobre el nivel de capacitación de las enfermeras en la atención a los pacientes con sida coincide con las necesidades de formación de los médicos, puesta de manifiesto en otros estu$\operatorname{dios}^{27-29}$. Las enfermeras se perciben con menor nivel de capacitación en el componente psicosocial de su práctica profesional (establecer una buena comunicación con el paciente y dar respuesta a las reacciones emocionales). Esta situación debería tenerse en cuenta a la hora de establecer los programas y estrategias de formación dirigidos a los profesionales de atención primaria.

A pesar de que la mayoría de las enfermeras se sienten capacitadas para la atención a los pacientes con alta precoz, cáncer y demencia, consideran que la formación recibida ha sido insuficiente para la atención de los pacientes con cáncer terminal, demencia y sida.

Del estudio podemos concluir que las enfermeras que realizan atención domiciliaria en Andalucía muestran niveles altos de satisfacción con el trabajo en atención primaria y con la atención domiciliaria a los grupos estudiados, con unos niveles de satisfacción más bajos en el caso de los pacientes con sida en fase terminal.

La mayoría de las enfermeras valoran a la familia como la principal fuente de bienestar para el enfermo en el ámbito domiciliario.

Se muestran favorables a la atención en el domicilio de pacientes con alta precoz, con demencia y cáncer. En el caso de pacientes con sida en fase ter- minal, la mitad de las enfermeras, si fuera posible, elegiría a otro tipo de paciente.

Encuentran dificultades en la atención domiciliaria de pacientes con cáncer y sida en fase terminal y entienden que la atención a estos enfermos requiere apoyo desde la atención especializada, lo que no ocurre en los pacientes con demencia y de alta precoz.

La valoración de la problemática social y dar respuestas a los problemas emocionales del paciente y familia es considerado por las enfermeras como parte integrante de su trabajo en atención domiciliaria.

Las enfermeras valoran el trabajo en equipo como un elemento clave en la evolución del paciente en el ámbito domiciliario, y consideran que sus conocimientos y habilidades tienen una gran influencia en la calidad de vida y la evolución de los enfermos.

La mayoría de las enfermeras se sienten capacitadas para la atención a los pacientes con alta precoz, siendo menor el número de las que se sienten capacitadas para la atención a pacientes con cáncer, demencia y sida.

El componente psicosocial de su práctica profesional (establecer una buena comunicación con el paciente y dar respuesta a las reacciones emocionales) constituye el ámbito en el que las enfermeras se perciben con un menor nivel de capacitación.

Agradecimientos. A todas/os las/os enfermeras/os de los centros de salud de Andalucía que han participado desinteresadamente en este proyecto y a todos los que se esfuerzan cada día por mejorar la atención domiciliaria que reciben sus pacientes.

\section{Bibliografía}

1. SESPAS. La salud y el sistema sanitario en E spaña. Informe SESPAS, 1995. Barcelona: SG E ditores, 1995.

2. Servicio Andaluz de Salud. Cartera de servicios de atención primaria 2000. Sevilla: SAS, Consejería de Salud, J unta de Andalucía, 2000.

3. Atención domiciliaria: una necesidad creciente [editorial]. Nursing 1996: 5.

4. Hernández PM, Rubio GA, Navarro E, Benítez MA, González G. Propuesta de un modelo de organización de atención domiciliaria basada en Atención Primaria. Cuadernos de Gestión 1999:5:31-8.

5. Pérez M, Palau N. Programas y servicios comunitarios de atención: viabilidad del lema "envejecer en casa". Rev Esp Geriatr Gerontol 1995;30:153-6.

6. Gené J, Contel J C. Propuesta para desarrollar la atención domiciliaria [editorial]. Aten Primaria 1999;23:15-20.

7. Frías $A$, Prieto $M A$, Heierle $C$, et al. La práctica enfermera en atención domiciliaria: perfil sociodemográfico y práctica profesional. Aten Primaria 2002;29:495-501.

8. Servicio Andaluz de Salud. Contrato programa del Servicio Andaluz de Salud con los Distritos de Atención Primaria. Sevilla: Servicio Andaluz de Salud, Consejería de Salud, J unta de Andalucía, 1997-2001.

9. Rosenstock I, Strecher VJ, Becker M. Social searning theory and the health belief model. Health Education Quarterly 1988;15:175-83.

10. Bandura A. Self-efficacy: toward a unifyng theory of behavioural change. Psychol Rev 1977;84:191-215.

11. Angladam N, Cañadell E. Satisfacción de enfermería de atención primaria en las comarcas del Alt y Baix Empordá. Aten Primaria 2000; 25:326-30

12. Fernández JI, Omínelo A, Villanueva A, Andrade C, Rivera M, Gómez $\mathrm{J} M$, et al. Satisfacción laboral de los profesionales de atención pri- 
maria del Área 10 del INSALUD de Madrid. Rev Esp Salud Pública 2000;74:139-47.

13. Acámer F, López C, López-Torres J. Satisfacción laboral de los profesionales sanitarios en atención primaria. Aten Primaria 1997; 20: 401-7.

14. Fernández JI, Villagrasa JR, Gamo MF, Vázquez J, Cruz E, Aguirre $M V$, et al. Estudio de la satisfacción laboral y sus determinantes en los trabajadores sanitarios de un Área de Madrid. Rev Esp Salud Pública 1995;69:487-97.

15. García A, Bermejo C, Mejías F. Satisfacción profesional de las enfermeras. Un estudio en el área 10 del INSALUD de Madrid. Rev Rol Enferm 1996;219:38-42.

16. Van-Der CJ, Baena C, Sánchez A, Mira JJ . Organización del trabajo de enfermería en atención primaria y satisfacción y estrés laboral: enfermería comunitaria frente a enfermería por programas. Centro de Salud 1995;757-61.

17. Cruz E, Vázquez J, Aguirre V, Fernández MI, Villagrasa JR, Andrada V. Evaluación de la satisfacción del personal de enfermería. Aten Primaria 1994;13:469-73.

18. Vidal C, Artigas B, Gogorcena MA, Galla J, Alorda C. Satisfacción laboral en atención primaria. Rev Rol Enferm 1993;176:13-7.

19. Puente A, Ribas A, Salla RM, Parrilla L, Espinás J, Caballé E, et al. Las demencias en el ámbito de la atención primaria. Aten Primaria 1997:19:419-22.

20. García S, Lekube D, Andikoetxean A, Solar M, Olaskoaga A. Características socioeconómicas, problemas y necesidades de salud de los pacientes crónicos domiciliarios. Aten Primaria 1997;20:230-6.
21. Durán MA. Los costes invisibles de la enfermedad. Bilbao: Fundación BBV, 1999

22. Prieto MA, Aceijas $C$, Heierle C, Frías A. Diseño y validación de un cuestionario sobre la práctica profesional enfermera en atención domiciliaria [en prensa].

23. Argimon j M, Jiménez J. Métodos de investigación. A plicados a la atención primaria de salud. Barcelona: Doyma, 1991.

24. Magallón R, Antoñanzas A. Medición de la calidad: recogida, análisis y presentación de los datos. En: Saturno P, Gascon JJ, Parra P, editores. Tratado de calidad asistencial en atención primaria. Tomo II. Madrid: Du Pont Pharma, 1997; p. 137-67.

25. López LA, J iménez J M, Luna J, Solas 0 , Martínez JI, Pérez MJ, et al. Sources of influence medical practice. J Epidemiol Community Health 2000;54:623-30.

26. Benítez MA, Salinas A, Asensio A, Armas J. Cuidados paliativos en atención primaria: opinión de los profesionales. Aten Primaria 1999;23:23-31.

27. Suárez-Varela J F, Zunzunegui MV, Binbela J L, Vilches A. Barreras de actitud y estructurales detectadas en médicos de atención primaria para atender a los pacientes con la infección VIH/SIDA. Aten Primaria 1997;20:62-70.

28. Contreras A, Clavero G, Casas G. Barreras de comunicación con el paciente VIH + percibidas por el personal sanitario. Gac Sanit 1995;9:91100.

29. Figueiras A, López-Álvarez XL, Monteagudo-Romero J, Taboada-Rodríguez XA. VIH/SIDA en atención primaria: opiniones y necesidades de los profesionales sanitarios gallegos. Aten Primaria 1997;20:93-9. 